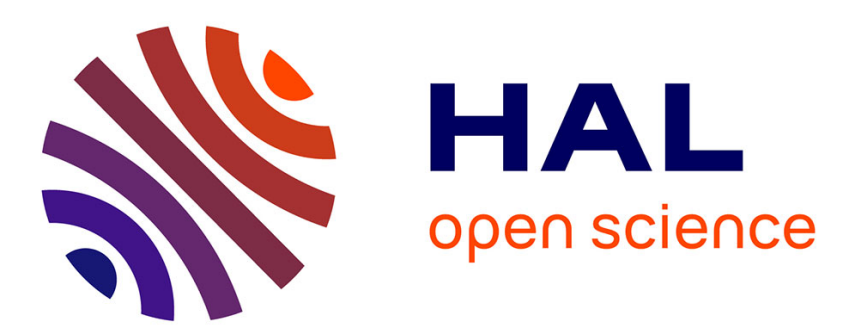

\title{
Electron energy-loss magnetic chiral dichroism of magnetic iron film affected by an underlayer in a double-layer structure
}

X. Fu, K. Wu, V. Serin, Bénédicte Warot-Fonrose, Q. He, R. Yang, L. Zhang, X. Huang

\section{To cite this version:}

X. Fu, K. Wu, V. Serin, Bénédicte Warot-Fonrose, Q. He, et al.. Electron energy-loss magnetic chiral dichroism of magnetic iron film affected by an underlayer in a double-layer structure. Applied Physics Letters, 2019, 115 (11), pp.112401. 10.1063/1.5100245 . hal-02349164

\section{HAL Id: hal-02349164 \\ https://hal.science/hal-02349164}

Submitted on 6 Nov 2020

HAL is a multi-disciplinary open access archive for the deposit and dissemination of scientific research documents, whether they are published or not. The documents may come from teaching and research institutions in France or abroad, or from public or private research centers.
L'archive ouverte pluridisciplinaire HAL, est destinée au dépôt et à la diffusion de documents scientifiques de niveau recherche, publiés ou non, émanant des établissements d'enseignement et de recherche français ou étrangers, des laboratoires publics ou privés. 


\title{
Electron energy-loss magnetic chiral dichroism of magnetic iron film affected by an underlayer in a double-layer structure
}

\author{
Cite as: Appl. Phys. Lett. 115, 112401 (2019); doi: 10.1063/1.5100245 \\ Submitted: 16 April 2019 - Accepted: 26 August 2019 . \\ Published Online: 9 September 2019 \\ X. Fu, ${ }^{1, \text { a) }}$ K. Wu, ${ }^{7}$ V. Serin, ${ }^{2}$ B. Warot-Fonrose, ${ }^{2}$ Q. He, ${ }^{7}$ R. Yang, ${ }^{7}$ L. Zhang, ${ }^{7}$ and X. Huang \\ AFFILIATIONS \\ ${ }^{7}$ College of Materials Science and Engineering, Chongqing University, Chongqing, 400044, China \\ ${ }^{2}$ Univ Toulouse, CEMES-CNRS UPR 8011, F-31055 Toulouse, France \\ a)xiaoxiao.fu@outlook.com
}

\begin{abstract}
The electron energy-loss magnetic chiral dichroism (EMCD) technique has been generally applied to single-phase magnetic crystals while rarely used for composite structures. It is mainly due to the lack of in-depth understanding of EMCD in the latter case where an additional phase may present under or above the investigated magnetic phase in the electron beam path. Here, we report EMCD signals acquired on a 15-nm-thick magnetic iron film with different thicknesses of the $\mathrm{MgO}$ substrate underlayer. By comparison, for areas with total thicknesses of $t=0.59 \lambda$ and $t=1.02 \lambda$ expressed with the mean free inelastic path of electron $\lambda$, the relative dichroic signals at the Fe- $L_{3}$ edge are $3.8 \% \pm 1.0 \%$ and $3.5 \% \pm 1.6 \%$, respectively, demonstrating no significant difference within the error range. However, the dichroic signal intensity at the Fe- $L_{2}$ edge peak is $77.6 \%$ larger in the thinner area of $t=0.59 \lambda$. Accordingly, the extracted $m_{L} / m_{s}$ ratio of Fe $3 d$ moments is $63 \%$ smaller in the thinner area even after the plural scattering is removed. Then, we confirm that the presence of an additional nonmagnetic phase under a magnetic iron crystal can noticeably affect the quantified value of the $m_{L} / m_{s}$ ratio of iron moment determined from the EMCD measurements. Furthermore, the larger thickness of the underlayer may result in relatively higher valuation of the $m_{L} / m_{s}$ ratio of the upper layer. A correction method, considering the different influence of the underlayer on the Fe- $L_{3}$ and $L_{2}$ edges, is in demand for developing potential applications of the EMCD technique to such composite nanomaterial systems.
\end{abstract}

Published under license by AIP Publishing. https://doi.org/10.1063/1.5100245

Electron energy-loss magnetic chiral dichroism (EMCD) is an electron energy-loss spectroscopy (EELS)-based technique to locally characterize element-specific spin and orbital magnetic moments in crystalline materials. ${ }^{1-3}$ The magnetic information is obtained from the EELS spectra recorded at two different positions on the diffraction plane, where different combinations of electron scattering vectors give rise to left- and right-handed polarized virtual photons in analogy with circular polarized X-rays in the synchrotron-based X-ray magnetic circular dichroism (XMCD) technique. ${ }^{1}$ Since EMCD was first demonstrated by Schattschneider et al., ${ }^{1}$ much effort has been made to develop it into a routine magnetic characterization technique in the transmission electron microscope (TEM). ${ }^{4-14}$ Various EMCD experimental setups, for instance the energy spectrum imaging, ${ }^{4,5}$ double aperture $q-E$ mode, ${ }^{6}$ spatially resolved EELS mode, ${ }^{7}$ and scanning TEM, ${ }^{8,9}$ have been proposed to improve the signal to noise ratio and the spatial resolution. In addition, the signal interpretation regarding the magnetic moment quantification has been widely concerned. The EMCD signal is not only simply affected by the intrinsic magnetism but also modulated by the dynamic electron diffraction condition which is related to the crystal structure, crystal orientation, and specimen thickness. ${ }^{3,10,11}$ With a deep understanding of the dynamic diffraction, the quantification of element-specific and even site-specific orbital and spin moments has been experimentally realized. ${ }^{11,12}$ Moreover, the theoretical simulation of the relative EMCD signal in various diffraction conditions has been widely applied for optimizing the experimental conditions ${ }^{13,14}$ of EMCD or broadening its applications. ${ }^{15}$

For the moment, the interpretation of quantitative results extracted from the EMCD signal is essentially based on an assumption that only one single-phase crystal is present in the electron beam path for each acquisition. Some EMCD experiments in the literature are actually in accordance with the assumed condition. For instance, experiments were performed on a single crystal in polycrystalline materials of $\mathrm{Fe},{ }^{9} \mathrm{Co},{ }^{16} \mathrm{Ni},{ }^{16}$ or their oxides ${ }^{7,17}$ or on epitaxially grown thin layers of single-crystalline $\mathrm{Fe}, \mathrm{Mn}$, and their compounds observed from 
the cross-sectional view..$^{10,14,18,19}$ However, the assumption may fail if the EMCD technique is applied to other widely used systems such as core-shell structures, embedded nanoparticles, and multilayers observed along the out-of-plane direction. More than one material may present in the beam direction, considering that a complete removal of one component material in these composite structures may be difficult or possibly introduces changes in magnetic moments of other components. Accordingly, there will be many open questions, for instance, how to understand the dynamic electron diffraction effects in such systems and how under the crystal the upper one affects the EMCD signal of each other. Actually, there have been reports of EMCD signals acquired on similar models, i.e., plane-view TEM samples of Fe thin films with $\mathrm{GaAs}^{1}$ and $\mathrm{MgO}^{5,6}$ substrates, while there is little discussion about the influence of substrate underlayers on the dichroic signal of the Fe film.

In this letter, we performed EMCD experiments on a plane-view sample of a single-crystalline iron thin film on $\mathrm{MgO}(001)$ substrates. EMCD signals of $\mathrm{Fe}-L_{2,3}$ edges were acquired at two different areas where the electron beam passed through the same thickness of the iron layer but a different thickness of the $\mathrm{MgO}$ underlayer. Comparisons were made on the EMCD signals and the corresponding orbital to spin moment ratios of $\mathrm{Fe} 3 d$ moment between the two areas. The influence of the $\mathrm{MgO}$ underlayer on the EMCD of the magnetic iron layer was discussed.

An iron thin film was deposited on a $\mathrm{MgO}(001)$ single crystal substrate via pulsed-laser deposition. Cross-sectional and plane-view TEM specimens were prepared with conventional methods including mechanical thinning (Multipre ${ }^{\mathrm{TM}}$ system) and $\mathrm{Ar}^{+}$ion-beam milling (PIPS-Gatan). The plane-view one, for instance, was first polished from the $\mathrm{MgO}$ substrate side until $10 \mu \mathrm{m}$ and then milled from the same side to achieve electron transparency. TEM experiments were performed at room temperature on a Tecnai F20 microscope operating at $200 \mathrm{kV}$ fitted with a postcolumn energy filter (Gatan GIF Quantum). The thickness uniformity and crystal structure of the iron layer were checked by bright field and high resolution TEM in the cross-sectional sample. EELS and EMCD experiments were performed on the plane-view sample. It should be noted that the plane-view sample was intentionally installed on the TEM holder in a way that the electron beam can first pass through the iron layer and then $\mathrm{MgO}$ under it. The ferromagnetic iron layer of the plane-view sample was constantly magnetized out-ofplane, in the direction parallel to the electron beam, to saturation by a magnetic field of $2 \mathrm{~T}$ from the objective lens in TEM (see the magnetic hysteresis curves in Fig. S1 in the supplementary material).

For EMCD experiments, a nearly parallel beam with a probe size of around $200 \mathrm{~nm}$ in diameter was used to illuminate the sample. Diffraction patterns were obtained in the conventional selected area electron diffraction (SAED) geometry. A $\pm(110)$ three-beam diffraction condition was achieved by tilting the Fe crystal by 5 degrees from the [001] zone axis. Two chiral positions, the symmetrical points on the Thales circle passing through the transmission and Fe(110) Bragg spots, were successively selected by the objective aperture. Accordingly, two EELS signals at Fe- $L_{2,3}$ edges included in the energy range from $650 \mathrm{eV}$ to $854 \mathrm{eV}$ were recorded, and normalization of the two signals was performed by aligning the integrate values under them in the range from $760 \mathrm{eV}$ to $800 \mathrm{eV}$. Orbital to spin moment ratios of iron along the electron beam axis were extracted from EMCD signals, which are the difference of the two EELS signals, with EMCD Sum Rules ${ }^{3}$ using Matlab. Statistical results were obtained by repeatedly acquiring
EMCD signals at the same experimental condition. More detailed information about the principle and experimental setups of EMCD are presented in Fig. S2 in the supplementary material. Meanwhile, lowloss EELS spectra were also acquired without any objective aperture, for checking the relative thickness $t / \lambda$ of the observed area and the plasmon scattering, where $t$ is the local thickness and $\lambda$ is the mean free inelastic path of the electron. The same experimental process was applied to two different areas, and their experimental results were compared with each other and also with the theoretical result simulated with a program demonstrated in Ref. 20. This program, on the basis of the simulation of elastic and inelastic electron scattering, can be used to simulate the dependence of the relative EMCD signal of the singlecrystalline sample on various experimental parameters such as sample tilt and sample thickness. ${ }^{20}$

Figure 1(a) presents a bright field TEM image of the cross section $\mathrm{Fe} / \mathrm{MgO}(001)$ sample observed from the $\mathrm{MgO}[010]$ zone axis. The thickness of the iron layer is quite uniform, which is measured to be $15 \mathrm{~nm}$. The SAED pattern of the area in the dashed circle of Fig. 1(a) is presented in Fig. 1(b), indicating that the iron layer is single crystalline and its epitaxial relationship with the $\mathrm{MgO}$ substrate is $\mathrm{Fe}[110](001) / /$ $\mathrm{MgO}[100](001)$. A high resolution TEM image of the Fe/MgO interface in Fig. 1(c) verifies that the iron layer was well epitaxially grown on the substrate. In this image, only $\mathrm{Fe}(110)$ lattice planes are resolved for the iron layer and both $\mathrm{MgO}(002)$ and (200) planes are resolved for the substrate. The $\mathrm{Fe}(110)$ interplanar spacing is measured to be
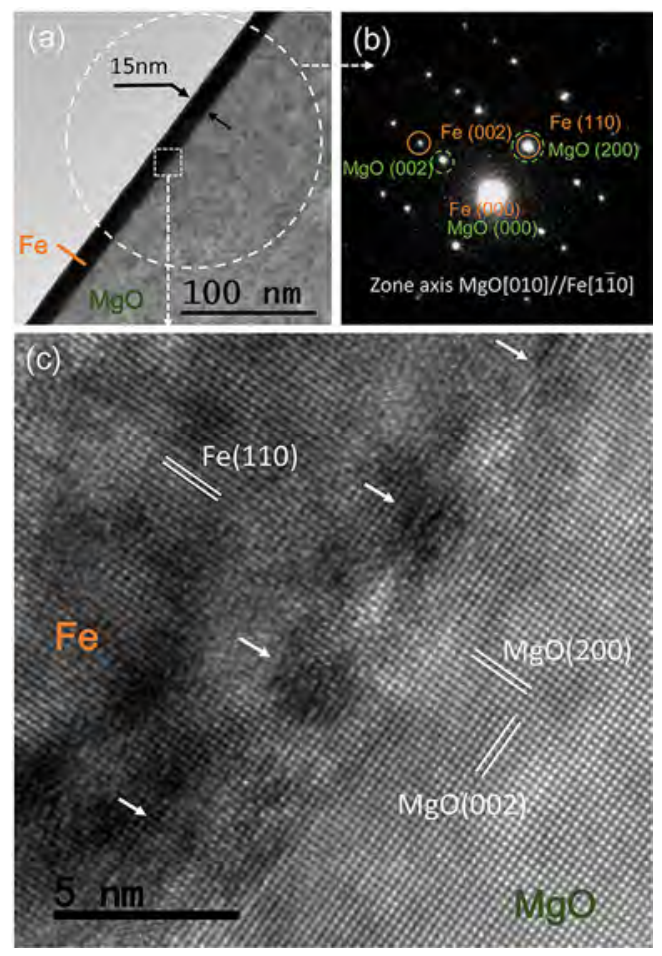

FIG. 1. (a) A bright field TEM image of the cross-sectional iron thin film on the $\mathrm{MgO}(001)$ substrate along the $\mathrm{MgO}[010] / / \mathrm{Fe}[1 \overline{1} 0]$ zone axis. (b) The diffraction pattern of the area in the dashed circle in (a). (c) The high resolution TEM image of the area in the dotted square in (a). 
$d_{\mathrm{Fe}(110)}=0.2028 \mathrm{~nm},{ }^{21}$ about $4 \%$ smaller than the $\mathrm{MgO}(002)$ interplanar spacing of $d_{\mathrm{MgO}(200)}=0.2110 \mathrm{~nm}^{22}$ The tensile stress of $\mathrm{MgO}$ on the Fe lattices has been released at the interface through the lattice mismatch dislocations, which introduce the contrasts indicated by white arrows in Fig. 1(c). Additionally, there is interdiffusion of Fe atoms into the $\mathrm{MgO}$ substrate, in the variation of $~ 3.5 \%$ along the interface. More information on the interface can be seen in Fig. S3 in the supplementary material.

Figure 2 demonstrates the experimental results of electron diffraction and low-loss EELS on the plane-view sample of $\mathrm{Fe} / \mathrm{MgO}(001)$. Figure 2(a) shows a conventional low-magnification TEM image. The areas of about $200 \mathrm{~nm}$ in diameter in the two circles marked as A and $\mathrm{B}$ are selected to be observed. Area A is closer to the vacuum area than area $\mathrm{B}$. The curvilinear contrasts that are most obvious near the vacuum are bend contours, and the areas in $\mathrm{A}$ and $\mathrm{B}$ are considered as flat without bending. Figure 2(b) compares the low-loss EELS spectra acquired at the two areas. Their zero-loss peak intensities have been normalized to 1 and centered at $0 \mathrm{eV}$. Both spectra show plasmon peaks approximately at the energy loss of $22 \mathrm{eV}$ with the full width at half maximum above $18 \mathrm{eV}$, while the peak height for area B is $45 \%$ larger than that for area A. Determined with a log-ratio method ${ }^{23}$ from the low-loss spectra intensity, the thickness $t=1.02 \lambda$ of area $\mathrm{B}$ is larger than $t=0.59 \lambda$ of area $\mathrm{A}$. The absolute values of the thickness in areas A and B are estimated to be around $60 \mathrm{~nm}$ and $100 \mathrm{~nm}$. Figs. 2(c) and 2 (d) show the corresponding diffraction patterns acquired at areas $\mathrm{A}$ and $\mathrm{B}$ along the zone axis of $\mathrm{Fe}[001] / / \mathrm{MgO}[001]$. It can be seen that two sets of diffraction spots are resolved in both Figs. 2(c) and 2(d).
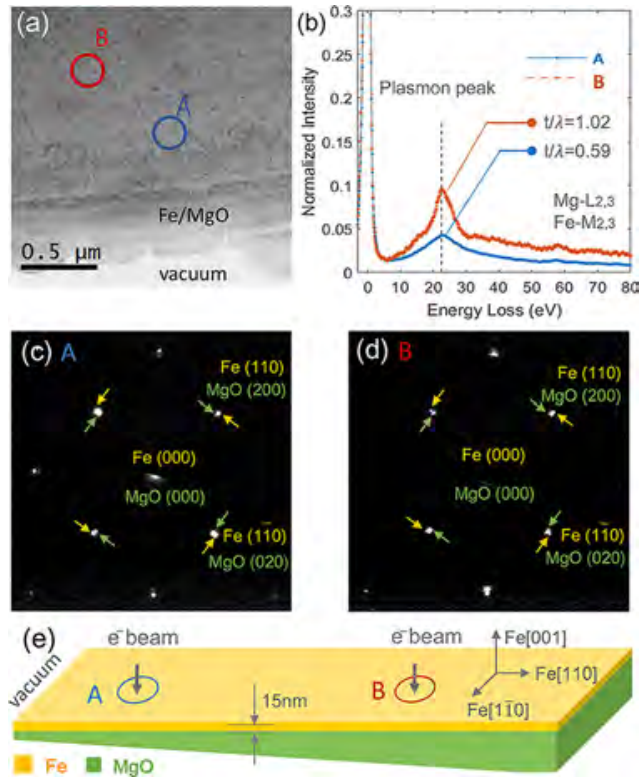

FIG. 2. (a) A conventional low-magnification TEM image of the $\mathrm{Fe} / \mathrm{MgO}(001)$ plane-view sample, in which area $A$ and area $B$ are marked with blue and red circles, respectively. (b) The low-loss EELS spectra of area $A$ and area $B$ with the zero-loss peak normalized to 1 and centered at $0 \mathrm{eV}$; the diffraction pattern acquired in (c) area $\mathrm{A}$ and (d) area $\mathrm{B}$ along the $\mathrm{MgO}(001) / / \mathrm{Fe}(001)$ zone axis and the yellow and green arrows indicate the diffraction spots of $\mathrm{Fe}$ and $\mathrm{MgO}$, respectively. (e) $A$ sketch to demonstrate the difference of areas $A$ and $B$ in the relative thickness of $\mathrm{Fe}$ and $\mathrm{MgO}$ crystals along the electron beam.
We index the patterns and ensure that one set of spots marked by yellow arrows are from the body-centered cubic (bcc) Fe crystal, and the other ones marked by green arrows are from face-centered cubic ( $\mathrm{fcc}$ ) $\mathrm{MgO}$. The distances from spot $\mathrm{Fe}(110)$ and $\mathrm{MgO}(200)$ to the transmission spot 0 have a $\sim 4 \%$ difference, so as the distance from $\mathrm{Fe}(1 \overline{1} 0)$ and $\mathrm{MgO}(002)$. It agrees with the difference of $d_{\mathrm{Fe}(110)}$ and $d_{\mathrm{MgO}(200)}$ interplanar spacings mentioned above. Thus, we ensure that areas $\mathrm{A}$ and $\mathrm{B}$ are double-layer structures of $\mathrm{Fe}$ and $\mathrm{MgO}$, and the Fe lattice is relaxed on $\mathrm{MgO}$. Now, we can say that there is a 15-nm-thick singlecrystalline bcc Fe thin film with single-crystalline fcc $\mathrm{MgO}$ underlayers presenting in the electron beam path in both areas $\mathrm{A}$ and $\mathrm{B}$, and the $\mathrm{MgO}$ layer is relatively thinner in area A, as sketched in Fig. 2(e).

Before demonstrating the experimental EMCD results of the two areas, Fig. 3(b) shows the simulated thickness-dependent relative dichroic signal for a bcc Fe crystal orientated in a $\pm(110)$ three-beam
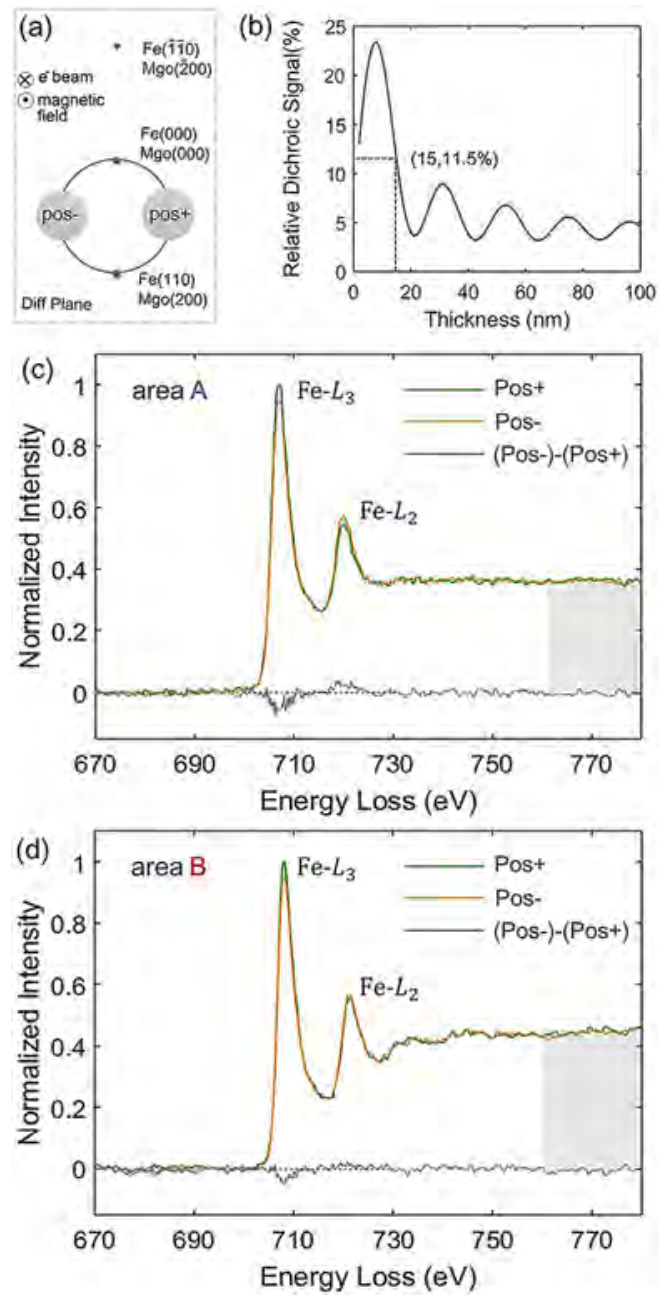

FIG. 3. (a) A sketch of the chiral positions in the diffraction plane. (b) The simulated relative dichroic signal of $\mathrm{Fe}-\mathrm{L}_{3}$ changing with the thickness of $\mathrm{Fe}$ in the $\pm(110)$ three-beam condition; the typical EELS and EMCD spectra (before the plural scattering removed) measured in (c) area $A$ and (d) area B, and the shadow areas indicate the normalization region. 
condition as shown in Fig. 3(a). The relative dichroic signal here is defined as the difference divided by the sum of the two chiral EELS spectra. We can see that the relative signal intensity fluctuates with the thickness of the Fe crystal due to dynamic electron diffraction effects, and the intensity is as high as $11.5 \%$ at the thickness of $15 \mathrm{~nm}$ which is the thickness of our magnetic iron film. It should be noted that the thickness in Fig. 3(b) does not include the thickness of other crystals as underlayers but only for the magnetic iron layer.

Typical experimental EELS and EMCD signals at $\mathrm{Fe}-\mathrm{L}_{2,3}$ edges of areas A and B are shown in Figs. 3(c) and 3(d). It can be seen in both (c) and (d) that the two EELS spectra acquired at chiral positions show an intensity difference at the Fe- $L_{3}$ and $\mathrm{Fe}-L_{2}$ edges, and the EMCD signals have opposite signs at the two edges. The noise level of the EMCD spectra, defined by the standard deviation of the EMCD signal in the pre-edge energy range divided by the average intensity of two EELS spectra at the Fe- $L_{3}$ peak, is $0.6 \%$ for area A and $1.0 \%$ for $\mathrm{B}$. The relative dichroic signal intensity at the $\mathrm{Fe}-L_{3}$ edge is measured to be $3.8 \% \pm 1.0 \%$ for area $\mathrm{A}$ and $3.5 \% \pm 1.6 \%$ for area $\mathrm{B}$. The error bar is determined from several EMCD spectra repeatedly acquired at the same area in the same experiment condition. Both experimental values are smaller than the theoretical value of $11.5 \%$. Actually, this discrepancy in similar material systems is generally presented in the literature. For instance, in Ref. 1, the value for a 10-nm-thick Fe thin film on the GaAs substrate of unknown thickness is measured to be 3.5\% (following our definition of the relative dichroic signal), smaller than its theoretical value of $16.1 \%$. Another experimental value is about $3.7 \%$ in Ref. 5 for a 10 -nm-thick Fe thin film on the $\mathrm{MgO}$ substrate of unknown thickness in a similar $\pm(110)$ three-beam condition as in our experiment. The information of the substrate thickness is not provided and discussed in these references. There are several possible causes of the relatively small experimental dichroic signal. First, the integration of the signal over the finite spectrometer entrance aperture and the nonzero convergence angle reduces the dichroic effect. ${ }^{1}$ Second, the electron beam passed through two phases which are bcc Fe and $\mathrm{fcc} \mathrm{GaAs}$ or $\mathrm{MgO}$. Their interface with imperfect dislocations and the difference in their lattice parameters make the real dynamic diffraction condition much different from the ideal condition assumed in the simulation and thus change the relative dichroic signal.

When comparing the two experimental values, no significant difference of the relative dichroic signal at the $\mathrm{Fe}-L_{3}$ edge for areas $\mathrm{A}$ and $\mathrm{B}$ is observed, given a $0.4 \%$ difference in their noise levels of EMCD signals and $0.6 \%$ difference in their error ranges. However, the EMCD signal intensity at the $\mathrm{Fe}-L_{2}$ edge peak is $77.6 \%$ lower for area B. Accordingly, the $m_{L} / m_{s}$ ratio of $\mathrm{Fe} 3 d$ moment calculated from the EMCD signals with EMCD sum rules is determined to be $0.11 \pm 0.02$ for area A with $t=0.59 \lambda$ and $0.24 \pm 0.03$ for area B with $t=1.02 \lambda$. The $m_{L} / m_{s}$ value for area $\mathrm{B}$ is $118 \%$ larger than the value for area $\mathrm{A}$, and both values are larger than 0.09 which is measured from a similar bcc iron layer with a thickness of $t=0.23 \lambda$ without the presence of the $\mathrm{MgO}$ substrate in Ref. 24. One cause of the discrepancy between the values for areas A and B may be the difference in plural scattering. In Figs. 3(c) and 3(d), we can easily see that the EELS spectra after the Fe- $L_{2}$ edge have higher intensity for area $B$ and especially the peak at the energy of around $730 \mathrm{eV}$ which is in accordance with the plasmon peak at $22 \mathrm{eV}$. Actually, the plasmon scattering has influence on the dichroic signal. ${ }^{24}$ For instance, it has been derived in Ref. 24 that the plural scattering has little influence on the dichroic signal intensity at the $\mathrm{Fe}-L_{3}$ edge but noticeably decreases the one at the $\mathrm{Fe}-L_{2}$ edge for bcc Fe, thus increasing the measured $m_{L} / m_{s}$ ratio of Fe $3 d$ moment. After removing the plasmon scattering by deconvoluting EELS spectra in Figs. 3(c) and 3(d) by low-loss EELS spectra in Fig. 2(b), the $m_{L} / m_{s}$ ratio is $0.08 \pm 0.02$ for area $A$ with $t=0.59 \lambda$ and $0.13 \pm 0.03$ for area B with $t=1.02 \lambda$. The $m_{L} / m_{s}$ value for area B is still $63 \%$ larger than the value for area A. Given the same thickness of the magnetic iron layer with saturated magnetization for the two areas, we confirm that the presence and the thickness variation of the $\mathrm{MgO}$ underlayer have a significant influence on the dichroic signal of the iron film and the extracted $m_{L} / m_{s}$ ratio of $\mathrm{Fe} 3 d$ moment. A larger underlayer thickness may results in higher valuation of the $m_{L} / m_{s}$ ratio. The results are contrary to the common understanding of the known EMCD sum rules, in which the dynamic diffraction effects and the intrinsic magnetism are two independent factors to influence the dichroic signal intensity, and the value of $m_{L} / m_{s}$ does not change with any variation of diffraction conditions. As to the cause of the contradictory, the possibility of different intrinsic magnetism for the two areas has been carefully ruled out by our previous discussion, and the dynamic electron diffractions for the case of double layers in the electron beam path should be concerned. It is possibly due to that the different influences of the dynamic diffractions in $\mathrm{MgO}$ underlayers of different thicknesses and the influence of the imperfect $\mathrm{Fe} / \mathrm{MgO}$ interface on the intensity of Fe- $L_{3}$ and $L_{2}$ edges have not been taken into consideration in the known sum rules. An investigation of a relative correction method is in demand.

In summary, we investigated the EMCD signal of a 15-nmthick magnetic iron film with a $\mathrm{MgO}$ substrate underlayer in the electron beam path. Two areas, $t=0.59 \lambda$ and $t=1.02 \lambda$ respectively, with the same thickness of iron but different thicknesses of $\mathrm{MgO}$ were selected and carefully checked with electron diffraction and low-loss EELS. The relative dichroic signals at the Fe- $L_{3}$ edge for the two selected areas present no significant difference within the error range, while they are more than three times smaller than the theoretical value that is simulated on a single-crystalline 15-nm-thick iron layer. In addition, the dichroic signal at the Fe- $L_{2}$ edge relative to the Fe- $L_{3}$ edge is smaller for the area of $t$ $=1.02 \lambda$ with a thicker $\mathrm{MgO}$ underlayer, and, correspondingly, the measured $m_{L} / m_{s}$ ratio is $63 \%$ larger. We thus experimentally verified that the presence of the layer under a magnetic iron film can affect the EMCD signal of iron and, more importantly, the extracted $m_{L} / m_{s}$ ratio of Fe $3 d$ moments, in a way that a larger underlayer thickness may result in higher valuation of the $m_{L} / m_{s}$ ratio. Our work provides important reference information for the potential application of the EMCD technique to composite nanomaterial systems.

See the supplementary material for the schematic diagram of the EMCD experimental setups, the magnetic hysteresis loops of our Fe/ $\mathrm{MgO}(001)$ sample, and the elemental map at its interface.

This work was supported by the National Natural Science Foundation of China (Grant No. 51701031); the State Key Research and Development Program of MOST, China (Grant No. 2016YFB0700400); and China Postdoctoral Science Foundation (Grant No. 2018M631060). We thank S. Löffler for providing the simulation program. 


\section{REFERENCES}

'P. Schattschneider, S. Rubino, C. Hébert, J. Rusz, J. Kuneš, P. Novák, E. Carlino, M. Fabrizioli, G. Panaccione, and G. Rossi, Nature 441, 486-488 (2006).

${ }^{2}$ D. Song, Z. Wang, and J. Zhu, Ultramicroscopy 201, 1-17 (2019).

${ }^{3}$ L. Calmels, F. Houdellier, B. Warot-Fonrose, C. Gatel, M. J. Hÿtch, V. Serin, E. Snoeck, and P. Schattschneider, Phys. Rev. B 76, 060409 (2007).

${ }^{4}$ B. Warot-Fonrose, F. Houdellier, M. J. Hÿtch, L. Calmels, V. Serin, and E. Snoeck, Ultramicroscopy 108, 393-398 (2008).

${ }^{5}$ B. Warot-Fonrose, C. Gatel, L. Calmels, V. Serin, and P. Schattschneider, Ultramicroscopy 110, 1033-1037 (2010).

${ }^{6} \mathrm{H}$. Ali, T. Warnatz, L. Xie, B. Hjörvarsson, and K. Leifer, Ultramicroscopy 196, 192-196 (2019)

${ }^{7}$ Z. Wang, A. H. Tavabi, L. Jin, J. Rusz, D. Tyutyunnikov, H. Jiang, Y. Moritomo, J. Mayer, R. E. Dunin-Borkowski, Y. Rong, and J. Zhu, Nat. Mater. 17, 221-225 (2018).

${ }^{8}$ S. Muto, J. Rusz, K. Tatsumi, R. Adam, S. Arai, V. Kocevski, P. M. Oppeneer,

D. E. Bürgler, and C. M. Schneider, Nat. Commun. 5, 3138 (2014).

${ }^{9}$ J. Rusz, S. Muto, J. Spiegelberg, R. Adam, K. Tatsumi, D. E. Bürgler, P. M. Oppeneer, and C. M. Schneider, Nat. Commun. 7, 12672 (2016).

${ }^{10} \mathrm{X}$. Fu, B. Warot-Fonrose, R. Arras, G. Seine, D. Demaille, M. Eddrief, V. Etgens, and V. Serin, Phys. Rev. B 93, 104410 (2016).
"Z. Q. Wang, X. Y. Zhong, R. Yu, Z. Y. Cheng, and J. Zhu, Nat. Commun, 4, 1395 (2013).

${ }^{12}$ D. Song, G. Li, J. Cai, and J. Zhu, Sci. Rep. 6, 18489 (2016).

${ }^{13}$ D. Song, J. Rusz, J. Cai, and J. Zhu, Ultramicroscopy 169, 44-54 (2016).

${ }^{14}$ G. Li, D. Song, Z. P. Li, and J. Zhu, Appl. Phys. Lett 108(24), 242414 (2016).

${ }^{15}$ D. Song, A. H. Tavabi, Z. A. Li, A. Kovács, J. Rusz, W. Huang, G. Richter, R. E. Dunin-Borkowski, and J. Zhu, Nat. Commun. 8, 15348 (2017).

${ }^{16}$ C. Hébert, P. Schattschneider, S. Rubino, P. Novák, J. Rusz, and M. StögerPollach, Ultramicroscopy 108, 277-284 (2008).

${ }^{17}$ Z. C. Wang, X. Y. Zhong, L. Jin, X. F. Chen, Y. Moritomo, and J. Mayer, Ultramicroscopy 176, 212-217 (2017).

${ }^{18}$ T. Thersleff, J. Rusz, B. Hjörvarsson, and K. Leifer, Phys. Rev. B 94, 134430 (2016).

${ }^{19}$ X. Fu, B. Warot-Fonrose, R. Arras, K. Dumesnil, and V. Serin, Phys. Rev. B 94, 140416 (2016).

${ }^{20}$ S. Löffler and P. Schattschneider, Ultramicroscopy 110, 831-835 (2010).

${ }^{21}$ Z. S. Basinski, W. Hume-Rothery, and A. L. Sutton, Proc. R. Soc. London, Ser. A 229(1179), 459-467 (1955).

${ }^{22} \mathrm{H}$. Bialas and K. Heneka, Vacuum 45(1), 79-87 (1994).

${ }^{23}$ R. F. Egerton, Electron Energy-Loss Spectroscopy in the Electron Microscope, 3rd ed. (Springer, Berlin, 2011).

${ }^{24}$ J. Rusz, H. Lidbaum, S. Rubino, B. Hjörvarsson, P. M. Oppeneer, O. Eriksson, and K. Leifer, Phys. Rev. B 83, 132402 (2011). 